\title{
Modelling and experimental investigation of geometrically graded shape memory alloys with parallel design configuration
}

\author{
Bashir S. Shariat*, Yinong Liu and Sam Bakhtiari \\ Department of Mechanical Engineering, The University of Western Australia, 35 Stirling \\ Highway, Perth, WA 6009, Australia \\ Email: bashir.samsamshariat@uwa.edu.au
}

\begin{abstract}
The shape memory effect and pseudoelastic effect of NiTi shape memory alloys occur within respectively a narrow temperature and stress window associated with its martensitic transformation. This renders difficulty in accurate and reliable control for actuation applications using these properties. One approach to improving controllability of an actuation component is to design a geometrically graded shape memory structure to create a nonuniform transformation field within the structure. This paper presents analytical modelling and experimental evaluation of geometrically graded NiTi structures with parallel design configuration. Closed-form solutions are obtained to describe the stress-strain relationship of such structures under tensile loading conditions, which can be used as an engineering tool for optimizing shape memory performances of such components. The geometrically graded structures exhibited partial stress gradient over stress-induced transformation. A maximum stress window of $420 \mathrm{MPa}$ was achieved over transformation stage, giving enlarged stress interval for shape memory actuation control.
\end{abstract}

Keywords: Shape memory alloy (SMA); NiTi; martensitic transformation; pseudoelasticity; functionally graded material (FGM); geometrical gradient.

\section{Introduction}

Shape memory alloys (SMAs) are a unique group of materials that have the ability to recover their original shapes from large deformations well beyond their elastic strain limits. The deformation recovery may occur spontaneously upon unloading or delayed until the material is heated to above a certain temperature $[1,2]$. The former behaviour is referred to as pseudoelasticity (or superelasticity) and the latter is referred to as the shape memory effect. 
These properties, which are unique among metal alloys, are associated with thermoelastic (i.e., reversible) martensitic transformations from an austenite (A) phase to a martensite (M) phase. These unique properties have rendered the alloys wide use in a range of engineering applications, such as sensors [3, 4], actuators [5, 6], and medical devices [7]. Some of these applications are reactive and require no precision of movement. Some other applications, such as mirror actuators [8], guidewires [9], and spinal rods [10], require precision and controllability of movement.

Among the various SMAs, NiTi-based alloys are by far the most widely used for their excellent properties. NiTi alloys exhibit a B2-B19' martensitic transformation. When induced by a tensile stress, the $\mathrm{B} 2 \rightarrow \mathrm{B} 19^{\prime}$ transformation often undergoes a Lüders-type deformation, which features a flat stress plateau with a nearly zero slope over a large transformation strain of $6 \sim 8 \%[11,12]$. This behaviour results in mechanical instability and poor control of displacement via application of load. This is a challenge for actuator design. A common approach to solving this problem is to design functionally graded (FG) NiTi. FG NiTi alloys provide expanded transformation stress (and temperature) windows, thus better controllability of the NiTi component $[13,14]$. In addition, FG NiTi may also exhibit new and unique properties, such as the "fishtail-like" behaviour of a compositionally or microstructurally graded NiTi thin strip $[15,16]$.

Fig. 1 shows the general design concept of functionally graded SMA structures. Based on coupling between the loading axis and the property gradient direction, the various designs can be classified into two basic types: the series design (Fig. 1(a)) and the parallel design (Fig. 1(b)). In series design configuration, the property gradient is along the loading direction. In parallel design configuration, the property gradient is perpendicular to the loading direction. In each design configuration, the property gradient can be obtained in three different ways: by variation in composition $[14,16]$, microstructure $[17,18]$, or geometry $[19,20]$.

In geometrically graded SMAs, the geometrical gradient causes the structure to experience a nonuniform stress field upon loading, thus progressive martensitic transformation within the structure $[21,22]$. This concept can be applied to various forms of the alloy, including sheets, plates, and rods $[19,20]$. In previous studies, we have investigated geometrically graded NiTi structures with gradients along the tensile loading axis (i.e., the series configuration) $[19,20$, 23] and developed analytical models for their deformation behaviour. This article presents the 
analytical modelling and experimental investigation of another design type with the geometrical gradient being perpendicular to the loading direction (i.e., the parallel configuration) as shown in Fig. 1(b)-(II). We used pseudoelastic NiTi material to create these FG structures.

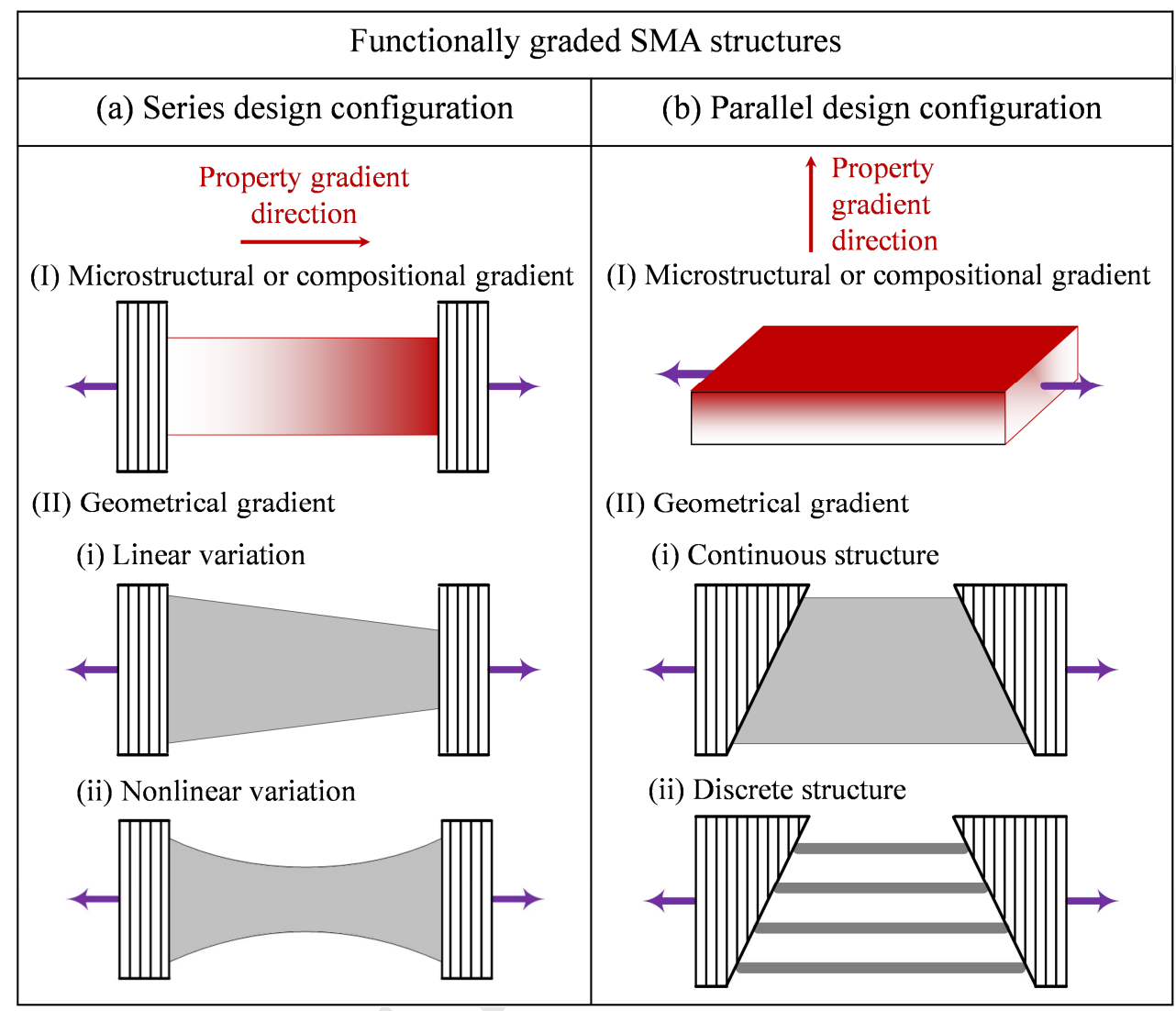

Fig. 1.General design concept of functionally graded SMA structures.

\section{Analytical modelling}

Fig. 2 shows the schematic drawing of a geometrically graded NiTi structure (based on the design concept of Fig. 1(b)-(II)-(ii)) and the definition of its material properties. $N$ parallel NiTi strips of width $w$, thickness $h$, and different lengths are arranged equidistantly and symmetrically along the $x$-axis, as illustrated in Fig. 2(a). The strip length varies linearly with respect to the $y$-axis from the minimum value $L_{1}$ to the maximum value $L_{2}$ corresponding to side (1) and side (2) of the structure, respectively. Therefore, the length of the $i$-th strip is obtained as

$$
L(i)=L_{1}+\frac{i-1}{N-1}\left(L_{2}-L_{1}\right)
$$


The length aspect ratio is defined as

$$
\alpha=L_{1} / L_{2}, \frac{1}{1+\frac{E_{A} \varepsilon_{t}}{\sigma_{t}}}<\alpha \leq 1
$$

The entire structure is under uniaxial tensile load $F$ along the $x$-axis. It is assumed that the gripping mechanism is rigid and avoids the sample rotation about any axes. As all strips have identical width and thickness, the nominal stress $\sigma$ is defined as the load over the total crosssectional area of the undeformed structure. Also, the nominal strain $\varepsilon$ is defined as the relative displacement of the grips along the loading direction $(\Delta L)$ over the initial maximum length $L_{2}$ :

$$
\begin{aligned}
& \sigma=\frac{F}{N w h} \\
& \varepsilon=\frac{\Delta L}{L_{2}}
\end{aligned}
$$

The material and transformation parameters are defined based on an ideal pseudoelastic behaviour of NiTi alloy under stress-induced martensitic transformation as shown in Fig. 2(b). $\sigma_{t}$ and $\sigma_{t}^{\prime}$ are forward and reverse transformation stresses, respectively. $\varepsilon_{t}$ and $\varepsilon_{t}^{\prime}$ are forward and reverse transformation strains, respectively. The apparent elastic moduli of the austenite and martensite are denoted as $E_{A}$ and $E_{M}$, respectively. $\sigma_{y}$ is the apparent yield stress of the material in the martensite phase. The reverse transformation strain $\mathcal{E}_{t}^{\prime}$ can be expressed in terms of the other independent transformation parameters from the geometrical relation found in Fig. 2(b), as

$$
\varepsilon_{t}^{\prime}=\varepsilon_{t}-\left(\frac{1}{E_{M}}-\frac{1}{E_{A}}\right)\left(\sigma_{t}-\sigma_{t}^{\prime}\right)
$$

The above parameters can be obtained for a pseudoelastic NiTi material through tensile experimentation. Fig. 2(c) shows the tensile deformation behaviour of a uniform Ti50.8 at $\% \mathrm{Ni}$ strip of $30 \times 1.5 \times 0.1 \mathrm{~mm}$ in dimension at $300 \mathrm{~K}$. The inset of this figure is the thermal transformation behaviour of the alloy as measured by differential scanning calorimetry (DSC). It reveals a two-step forward $\mathrm{A} \rightarrow \mathrm{R} \rightarrow \mathrm{M}$ and a one-step reverse $\mathrm{M} \rightarrow \mathrm{A}$ phase transformation, where $\mathrm{R}$ represents the trigonal phase of NiTi. The transformation temperatures are defined on the DSC curve. As evident in the DCS measurement, the 
transformation between $\mathrm{A}$ and $\mathrm{M}$ phases is the thermally-induced $\mathrm{A} \rightarrow \mathrm{R}$ transformation. The corresponding stress for inducing this transformation during deformation obeys ClausiusClapeyron equation, with a linear slope of $14 \mathrm{MPa} / \mathrm{K}$ [24]. Considering that the testing temperature (room temperature) is $\sim 25 \mathrm{~K}$ above the $\mathrm{A} \rightarrow \mathrm{R}$ transformation temperature, the stress required to induce the transformation at the room temperature is $14 \times 25=350 \mathrm{MPa}$. It is seen that the stress plateau of the stress-strain curve shown in Fig. 2(c) is at $<350 \mathrm{MPa}$. This means the $\mathrm{A} \rightarrow \mathrm{R}$ transformation is not induced but bypassed during the stress-induced $\mathrm{A} \rightarrow \mathrm{M}$ transformation.

The material properties of this alloy are specified according to Fig. 2(c), as

$$
\begin{aligned}
& \sigma_{t}=350 \mathrm{MPa}, \sigma_{t}^{\prime}=70 \mathrm{MPa}, \sigma_{y}=850 \mathrm{MPa} \\
& \varepsilon_{t}=0.062, \varepsilon_{t}^{\prime}=0.053 \\
& E_{A}=39 \mathrm{GPa}, E_{M}=17 \mathrm{GPa}
\end{aligned}
$$

It is noted that the above value of $E_{M}$ is obtained by considering the section of stress-strain curve between the end of plateau and the yield limit of martensite. Also, the above value of $E_{A}$ is appropriately selected to take into account a possible minor occurrence of the $\mathrm{A} \rightarrow \mathrm{R}$ transformation (prior to the stress plateau), which may manifest as a reduced apparent modulus of elasticity of the alloy.

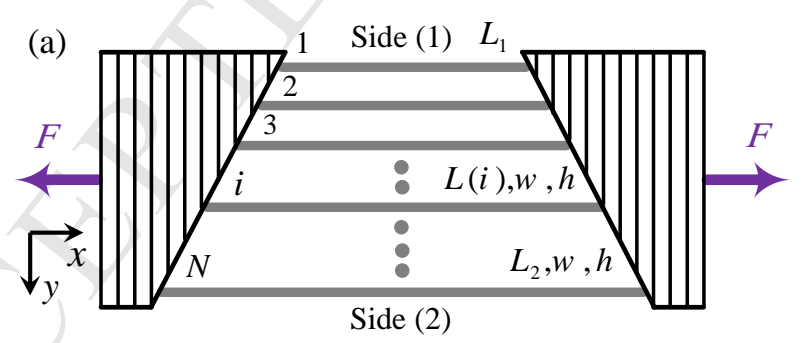



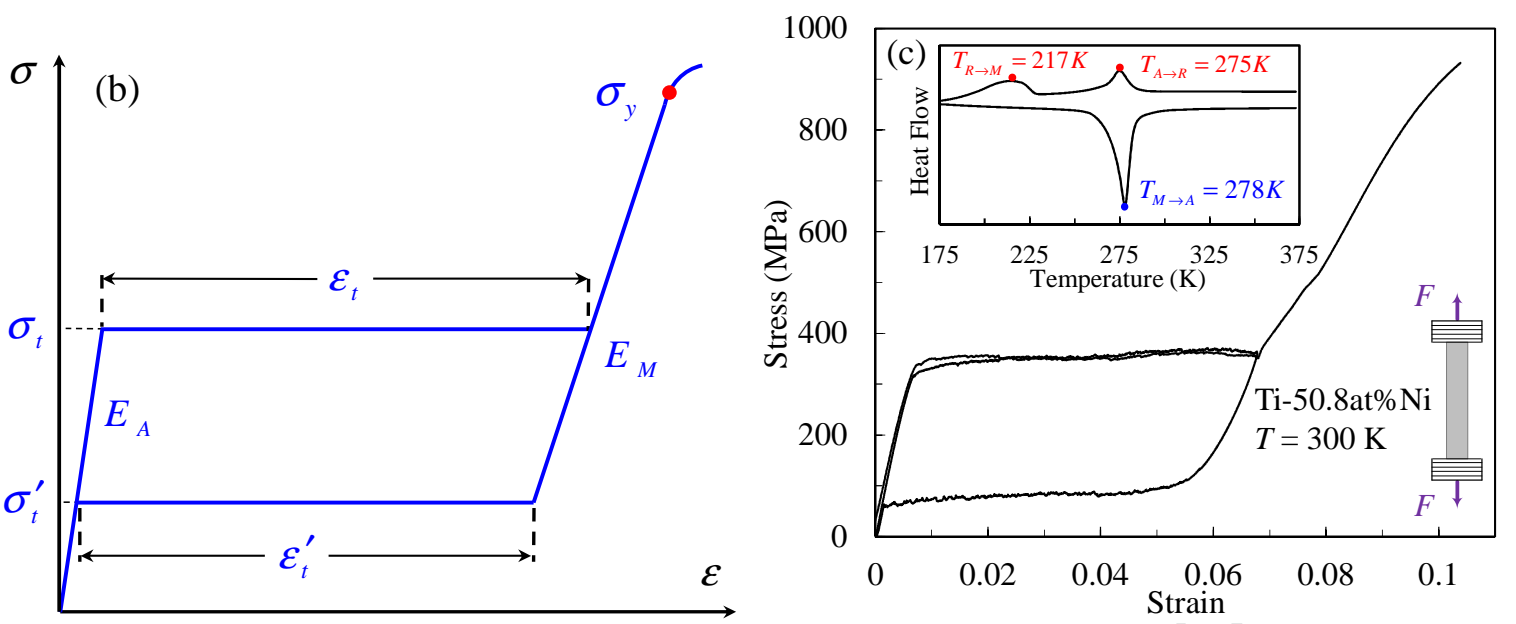

Fig. 2. The geometrically graded NiTi structure; (a): the schematic drawing of the parallelstrip system, (b): the definition of material parameters, (c): tensile testing of Ti-50.8at\% Ni alloy used for defining material properties.

Here, we establish the nominal stress-strain relationship of the geometrically graded NiTi structure with parallel design configuration under uniaxial tensile loading as shown in Fig. 2(a). Due to the involvement of the martensitic transformation, we need to consider separate stages of deformation to obtain the stress-strain relationship.

2.1. Stage (1): $0 \leq \varepsilon<\alpha \frac{\sigma_{t}}{E_{A}}$

At this stage, the entire structure is in the austenite phase since the applied load is less than the critical value to induce martensitic transformation in the shortest strip on side (1), which experiences the maximum strain, thus stress. The load $F$ is written as

$$
F=\sum_{i=1}^{N} E_{A} \frac{\Delta L}{L(i)} w h
$$

Using Eqs. (1)-(3) and (6), the nominal stress-strain relationship for this stage is obtained as

$$
\sigma=\frac{E_{A} \varepsilon}{N} \sum_{i=1}^{N} \frac{1}{\alpha+\frac{i-1}{N-1}(1-\alpha)}
$$

2.2. Stage (2): $\alpha \frac{\sigma_{t}}{E_{A}} \leq \varepsilon<\frac{\sigma_{t}}{E_{A}}$ 
Fig. 3(a) shows how transformation initiates within the structure. At this stage, the stressinduced martensitic transformation starts at the shortest strip on side (1), where the strain is the highest and the stress reaches the forward transformation stress $\sigma_{t}$ first. As the load increases, the transformation progressively propagates to the longer strips. This stage ends when the transformation starts at the longest strip on side (2). During this stage, the locus of the start of the $\mathrm{A} \rightarrow \mathrm{M}$ transformation moves across the transverse direction among the strips from side (1) to side (2), with the strips behind being partially transformed under the constant stress $\sigma_{t}$ (denoted $\left.\mathrm{A} \rightarrow \mathrm{M}\right)$ and those in front still in fully austenite state (denoted A). The movement (propagation) of the transformation front from strip to strip is defined by an integer parameter $T$, which varies from 1 to $N$ and denotes the last strip that has started transformation. At the moment when the $\mathrm{A} \rightarrow \mathrm{M}$ transformation reaches to the $T$-th strip, it can be written

$$
\sigma_{t}=E_{A} \frac{\Delta L}{L(T)}
$$

Using Eqs. (1)-(3) and (8), the nominal strain at the start of transformation in the $T$-th strip is found as

$$
\varepsilon=\frac{\sigma_{t}}{E_{A}}\left(\alpha+\frac{T-1}{N-1}(1-\alpha)\right)
$$

The load $F$ is divided into two parts carried by the partially transformed region and the fully austenite region:

$$
F=F_{A \rightarrow M}+F_{A}=\sigma_{t} T w h+\sum_{i=T+1}^{N} E_{A} \frac{\Delta L}{L(i)} w h
$$

Using Eqs. (1)-(3) and (10), the nominal stress-strain relationship for this stage is obtained as

$$
\sigma=\frac{1}{N}\left(\sigma_{t} T+E_{A} \varepsilon \sum_{i=T+1}^{N} \frac{1}{\alpha+\frac{i-1}{N-1}(1-\alpha)}\right)
$$

Eqs. (9) and (11) are the principal equations to define nominal strain intervals and corresponding nominal stress-strain relationships in stage (2). 

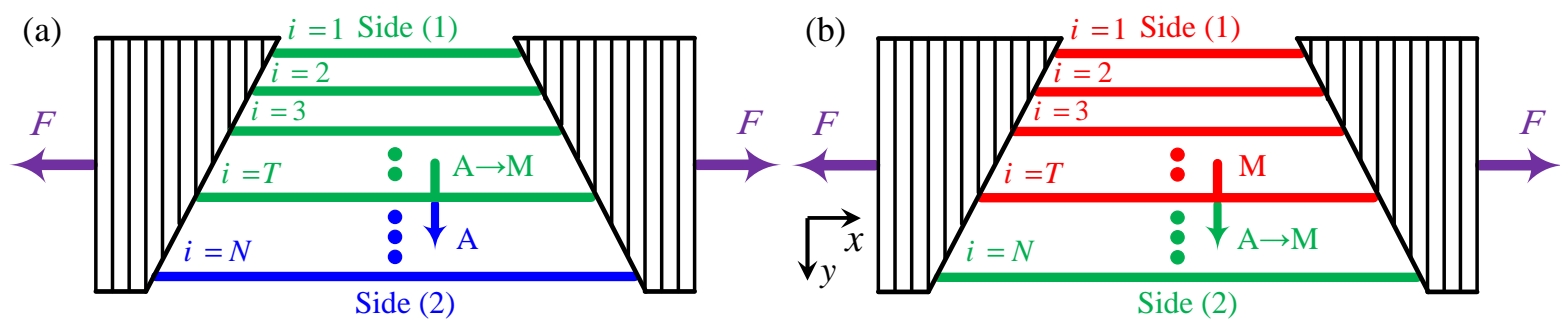

Fig. 3. Evolution of martensitic transformation within the geometrically graded NiTi structure; (a): initiation of transformation at stage (2), (b): completion of transformation at stage (4).

2.3. Stage (3): $\frac{\sigma_{t}}{E_{A}} \leq \varepsilon<\alpha\left(\frac{\sigma_{t}}{E_{A}}+\varepsilon_{t}\right)$

During this stage, all of the strips are in the course of martensitic transformation. Therefore, the overall load $F$ remains unchanged at the maximum (final) load of the previous stage, while the structure is nonuniformly stretched along the $x$-axis. The nominal stress during this stage is

$$
\sigma=\sigma_{t}
$$

This stage ends when the transformation is completed at the shortest strip on side (1), which has the maximum local strain level, while other strips are still in the course of transformation.

2.4. Stage (4): $\alpha\left(\frac{\sigma_{t}}{E_{A}}+\varepsilon_{t}\right) \leq \varepsilon<\frac{\sigma_{t}}{E_{A}}+\varepsilon_{t}$

This stage starts when the shortest strip on side (1) is fully transformed to martensite and ends when the longest strip has completed its martensitic transformation. As the load increases, the locus of the end of the transformation (i.e., the boundary between the fully martensite region $(\mathrm{M})$ and the partially transformed region $(\mathrm{A} \rightarrow \mathrm{M})$ ) moves gradually from side (1) toward side (2) as shown in Fig. 3(b). The movement of the boundary between these two regions is defined by an integer parameter $T$, which varies from 1 to $N$ and denotes the last strip that has fully transformed. At the moment when the $\mathrm{A} \rightarrow \mathrm{M}$ transformation has reached completion in the $T$-th strip, it can be written

$$
\sigma_{t}=E_{A}\left(\frac{\Delta L}{L(T)}-\varepsilon_{t}\right)
$$


Using Eqs. (1)-(3) and (13), the nominal strain at the completion of transformation in the T-th strip is found as

$$
\varepsilon=\left(\frac{\sigma_{t}}{E_{A}}+\varepsilon_{t}\right)\left(\alpha+\frac{T-1}{N-1}(1-\alpha)\right)
$$

The load $F$ is divided into two parts carried by the partially transformed region and the fully martensite region:

$$
F=F_{A \rightarrow M}+F_{M}=\sigma_{t}(N-T) w h+\sum_{i=1}^{T}\left[\sigma_{t}+E_{M}\left(\frac{\Delta L}{L(i)}-\frac{\sigma_{t}}{E_{A}}-\varepsilon_{t}\right)\right] w h
$$

Using Eqs. (1)-(3) and (15), the nominal stress-strain relationship for this stage is obtained as

$$
\sigma=\sigma_{t}-\frac{1}{N}\left[T\left(\frac{\sigma_{t}}{E_{A}}+\varepsilon_{t}\right)-E_{M} \varepsilon \sum_{i=1}^{T} \frac{1}{\alpha+\frac{i-1}{N-1}(1-\alpha)}\right]
$$

Eqs. (14) and (16) are the principal equations to define nominal strain intervals and corresponding nominal stress-strain relationships in stage (4).

2.5. Stage (5): $\varepsilon \geq \frac{\sigma_{t}}{E_{A}}+\varepsilon_{t}$

At this stage, all strips have fully transformed to martensite and are elastically stretched in the martensite phase. The load $F$ is written as

$$
F=\sum_{i=1}^{N}\left[\sigma_{t}+E_{M}\left(\frac{\Delta L}{L(i)}-\frac{\sigma_{t}}{E_{A}}-\varepsilon_{t}\right)\right] w h
$$

Using Eqs. (1)-(3) and (17), the nominal stress-strain relationship for this stage is obtained as

$$
\sigma=\sigma_{t}-E_{M}\left(\frac{\sigma_{t}}{E_{A}}+\varepsilon_{t}\right)+\frac{E_{M} \varepsilon}{N} \sum_{i=1}^{N} \frac{1}{\alpha+\frac{i-1}{N-1}(1-\alpha)}
$$

Up to now, the nominal stress-strain relationships of the geometrically graded NiTi structure consisting of $N$ parallel strips for the five stages of loading have been established. The same number of deformation stages can be defined for unloading. To define the strain interval of each reverse stage and the corresponding nominal stress-strain relationship, we can substitute $\sigma_{t}$ and $\varepsilon_{t}$ by $\sigma_{t}^{\prime}$ and $\varepsilon_{t}^{\prime}$ in the strain interval and nominal stress-strain relationship of the 
similar forward stage and use Eq. (4) to express the final relationships in terms of independent transformation parameters.

\subsection{Two-strip design}

Fig. 4(a) shows schematically a parallel-strip structure consisting two NiTi strips. By considering $N=2$, Eqs. (7), (9), (11), (12), (14), (16), and (18) are reduced to the principal equations to describe the stress-strain behaviour of this structure as summarised in Table 1 . Fig. 4(b) shows the nominal stress-strain diagram of the geometrically graded structure with $\alpha=0.7$ based on the analytical solution presented in Table 1 and the material properties specified in Eq. (5) (the red curve). The corresponding deformation stages are also defined on the stress-strain curve. The stress-strain loop shown in black lines represent the original deformation behaviour of the alloy (corresponds to $\alpha=1$ ). It is seen that the stress-strain curve of the geometrically graded structure partially deviates from the original stress-strain behaviour of the NiTi alloy. The $\mathrm{A} \rightarrow \mathrm{M}$ transformation in this structure occurs in stages (2), (3) and (4), creating a nonuniform "stress plateau" behaviour over a stress window. Using Table 1, the plateau length $(p l)$ of stage (3), the stress window $\left(\sigma_{w}\right)$, and the stress-strain slope $(s)$ of stage (4) are obtained as

$$
p l=\alpha \varepsilon_{t}, \sigma_{w}=\frac{(1-\alpha) E_{M}}{2 \alpha}\left(\frac{\sigma_{t}}{E_{A}}+\varepsilon_{t}\right), s=\frac{E_{M}}{2 \alpha}
$$

As understood from Eq. (19), by decreasing the length aspect ratio $\alpha$, the plateau length in stage (3) is decreased and the stress window and the stress-strain slope of stage (4) are increased. These variations can be observed in Fig. 4(c), which shows the deformation behaviour of the structures with different aspect ratios.

Table 1. Stress-strain relationship of a two-strip structure at different deformation stages.

\begin{tabular}{|l|l|l|}
\hline Stage & Nominal strain interval & Nominal stress-strain relationship \\
\hline 1 & $0 \leq \varepsilon<\alpha \frac{\sigma_{t}}{E_{A}}$ & $\sigma=\frac{E_{A} \varepsilon(\alpha+1)}{2 \alpha}$ \\
\hline 2 & $\alpha \frac{\sigma_{t}}{E_{A}} \leq \varepsilon<\frac{\sigma_{t}}{E_{A}}$ & $\sigma=\frac{\sigma_{t}+E_{A} \varepsilon}{2}$ \\
\hline 3 & $\frac{\sigma_{t}}{E_{A}} \leq \varepsilon<\alpha\left(\frac{\sigma_{t}}{E_{A}}+\varepsilon_{t}\right)$ & $\sigma=\sigma_{t}$ \\
\hline
\end{tabular}




\begin{tabular}{|l|l|l|}
\hline 4 & $\alpha\left(\frac{\sigma_{t}}{E_{A}}+\varepsilon_{t}\right) \leq \varepsilon<\frac{\sigma_{t}}{E_{A}}+\varepsilon_{t}$ & $\sigma=\sigma_{t}+\frac{E_{M}}{2 \alpha}\left[\varepsilon-\alpha\left(\frac{\sigma_{t}}{E_{A}}+\varepsilon_{t}\right)\right]$ \\
\hline 5 & $\varepsilon \geq \frac{\sigma_{t}}{E_{A}}+\varepsilon_{t}$ & $\sigma=\sigma_{t}-E_{M}\left(\frac{\sigma_{t}}{E_{A}}+\varepsilon_{t}\right)+\frac{E_{M} \varepsilon(\alpha+1)}{2 \alpha}$ \\
\hline
\end{tabular}
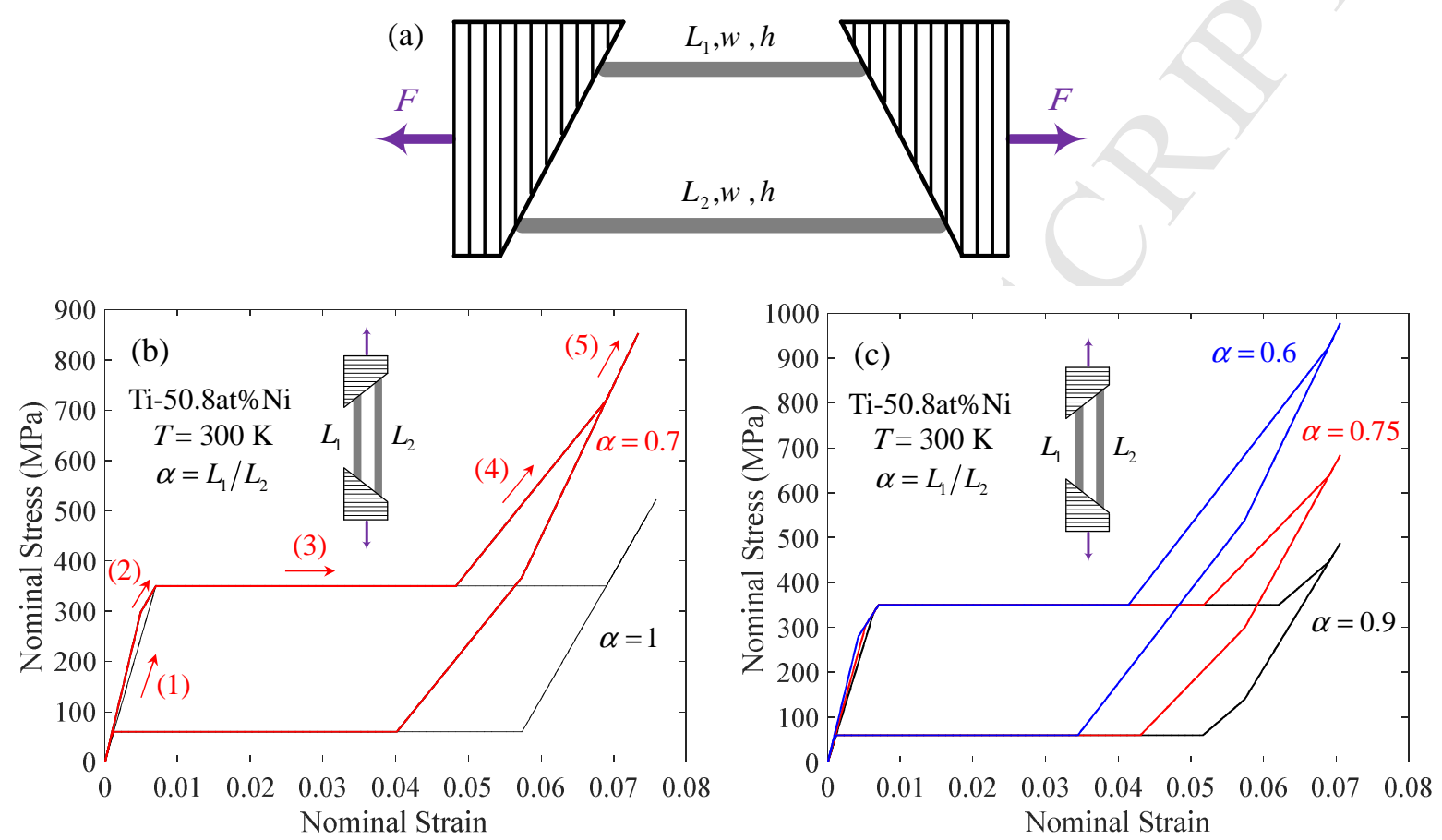

Fig. 4. Two-strip design of the geometrically graded NiTi structure, (a): schematic drawing of the structure, (b): the deformation behaviour for $\alpha=0.7$ and deformation stages, (c): the effect of $\alpha$ variation on the deformation behaviour.

\subsection{Three-strip design}

Fig. 5(a). shows schematically a parallel-strip structure consisting three NiTi strips. By considering $N=3$, Eqs. (7), (9), (11), (12), (14), (16), and (18) are reduced to the principal equations to describe the stress-strain behaviour of this structure as summarised in Table 2. Fig. 5(b) shows the nominal stress-strain diagram of such structure with $\alpha=0.7$ based on the analytical solution presented in Table 2 and the material properties specified in Eq. (5) (the red curve). The different deformation stages are also denoted on the stress-strain curve. It is seen that stage (4) consists of two substages with different slopes. Fig. 5(c) shows a comparison of the deformation behaviours of three designs, including a two-strip design, a three-strip design, and four-strip design, all with $\alpha=0.7$. It is seen that the main differences 
occur in stage (4) (and the corresponding unloading section prior to the lower stress plateau), with the number of substages corresponding to the number of strips. Also, all structures reach to the nearly same nominal stress level at $\varepsilon=0.07$.

Table 2. Stress-strain relationship of a three-strip structure at different deformation stages.

\begin{tabular}{|l|l|l|}
\hline Stage & Nominal strain interval & Nominal stress-strain relationship \\
\hline 1 & $0 \leq \varepsilon<\alpha \frac{\sigma_{t}}{E_{A}}$ & $\sigma=\frac{E_{A} \varepsilon}{3}\left(1+\frac{1}{\alpha}+\frac{2}{\alpha+1}\right)$ \\
\hline 2 & $\alpha \frac{\sigma_{t}}{E_{A}} \leq \varepsilon<\frac{(\alpha+1) \sigma_{t}}{2 E_{A}}$ & $\sigma=\frac{1}{3}\left[\sigma_{t}+E_{A} \varepsilon\left(1+\frac{2}{\alpha+1}\right)\right]$ \\
\hline 3 & $\frac{(\alpha+1) \sigma_{t}}{2 E_{A}} \leq \varepsilon<\frac{\sigma_{t}}{E_{A}}$ & $\sigma=\frac{1}{3}\left(2 \sigma_{t}+E_{A} \varepsilon\right)$ \\
\hline 4 & $\alpha\left(\frac{\sigma_{t}}{E_{A}}+\varepsilon_{t}\right) \leq \varepsilon<\left(\frac{\alpha+1}{2}\right)\left(\frac{\sigma_{t}}{E_{A}}+\varepsilon_{t}\right)$ & $\sigma=\sigma_{t}+\frac{E_{M}}{3}\left[\frac{\varepsilon}{\alpha}-\left(\frac{\sigma_{t}}{E_{A}}+\varepsilon_{t}\right)\right]$ \\
& $\left(\frac{\alpha+1}{2}\right)\left(\frac{\sigma_{t}}{E_{A}}+\varepsilon_{t}\right) \leq \varepsilon<\left(\frac{\sigma_{t}}{E_{A}}+\varepsilon_{t}\right)$ & $\sigma=\sigma_{t}+\frac{E_{M}}{3}\left[\varepsilon\left(\frac{1}{\alpha}+\frac{2}{\alpha+1}\right)-2\left(\frac{\sigma_{t}}{E_{A}}+\varepsilon_{t}\right)\right]$ \\
\hline 5 & $\varepsilon \geq \frac{\sigma_{t}}{E_{A}}+\varepsilon_{t}$ \\
\hline
\end{tabular}



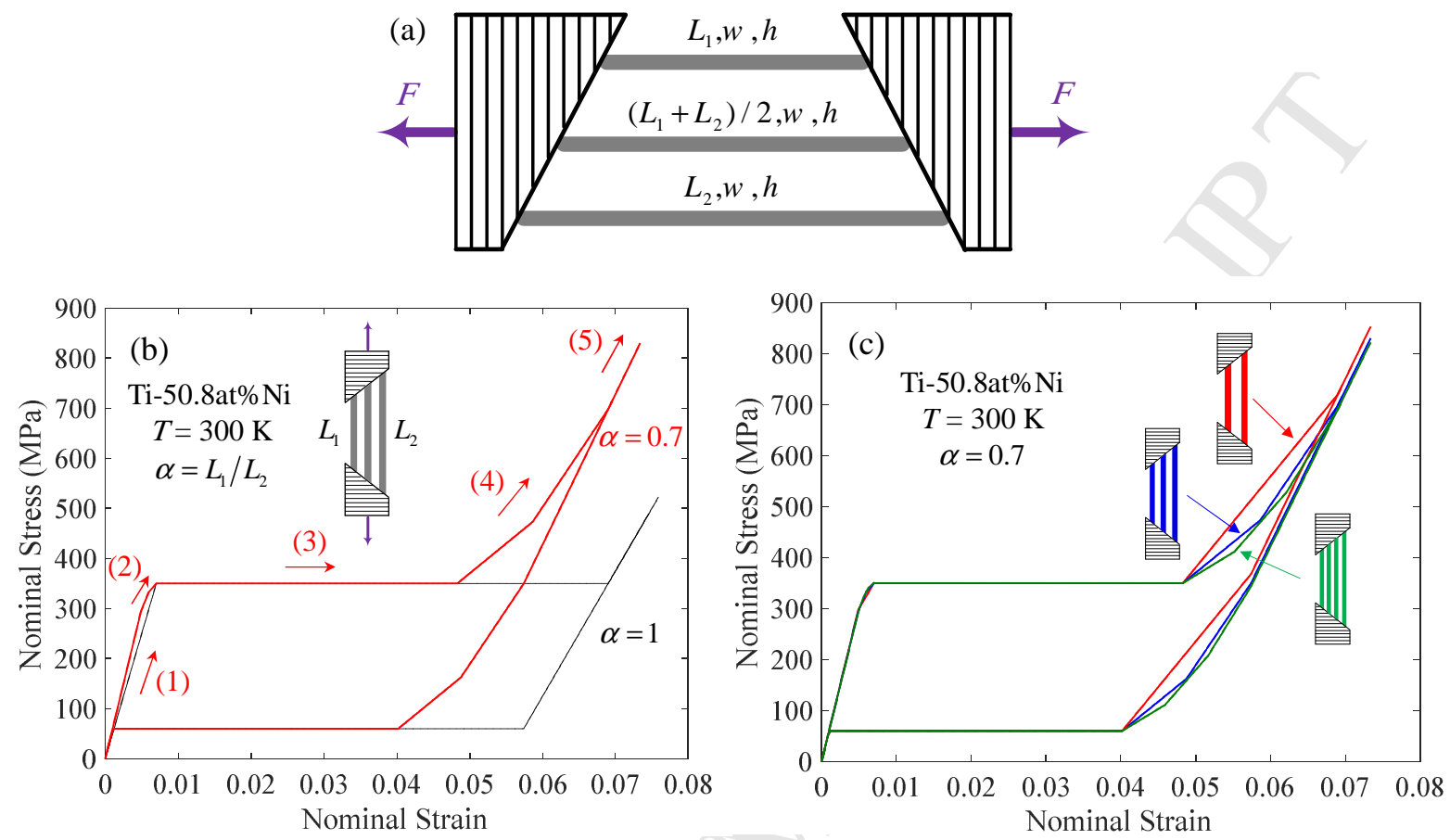

Fig. 5. Three-strip design of the geometrically graded NiTi structure, (a): schematic drawing of the structure, (b): the deformation behaviour for $\alpha=0.7$ and deformation stages, (c): the comparison of the deformation behaviour with that of two-strip and four-strip structures.

\subsection{Tapered plate design}

Fig. 6(a) shows schematically a geometrically graded NiTi plate (based on the design concept of Fig. 1(b)-(II)-(i)) with length aspect ratio $\alpha$, width $b$ and thickness $h$. The gradient is continuous and is created by a symmetrical taper at each end with respect to the $y$-axis. The tapered plate is under a tensile load $F$ along the $x$-axis. As the plate is strained more on side (1) than on side (2) at a given elongation, the martensitic transformation initiates at side (1) and gradually propagates toward side (2) as the load increases. Figs. 6(b) and 6(c) show how the $\mathrm{A} \rightarrow \mathrm{M}$ transformation propagates within the plate. The same analytical approach used for the parallel-strip structure in Sections 2.1 to 2.5 , can be applied to the tapered plate to establish stress-strain relationship under uniaxial tension. The details of the analytical process to derive the principal equations are provided in Appendix A. These equations are summarised in Table 3 for all deformation stages during loading. Using this table and the material properties specified in Eq. (5), the nominal stress-strain curve of the tapered plate 
with $\alpha=0.7$ is calculated, as plotted in Fig. 6(d). It is seen that stage (4) of the deformation is a nonlinear variation of stress versus strain unlike that in the discrete parallel-strip designs.
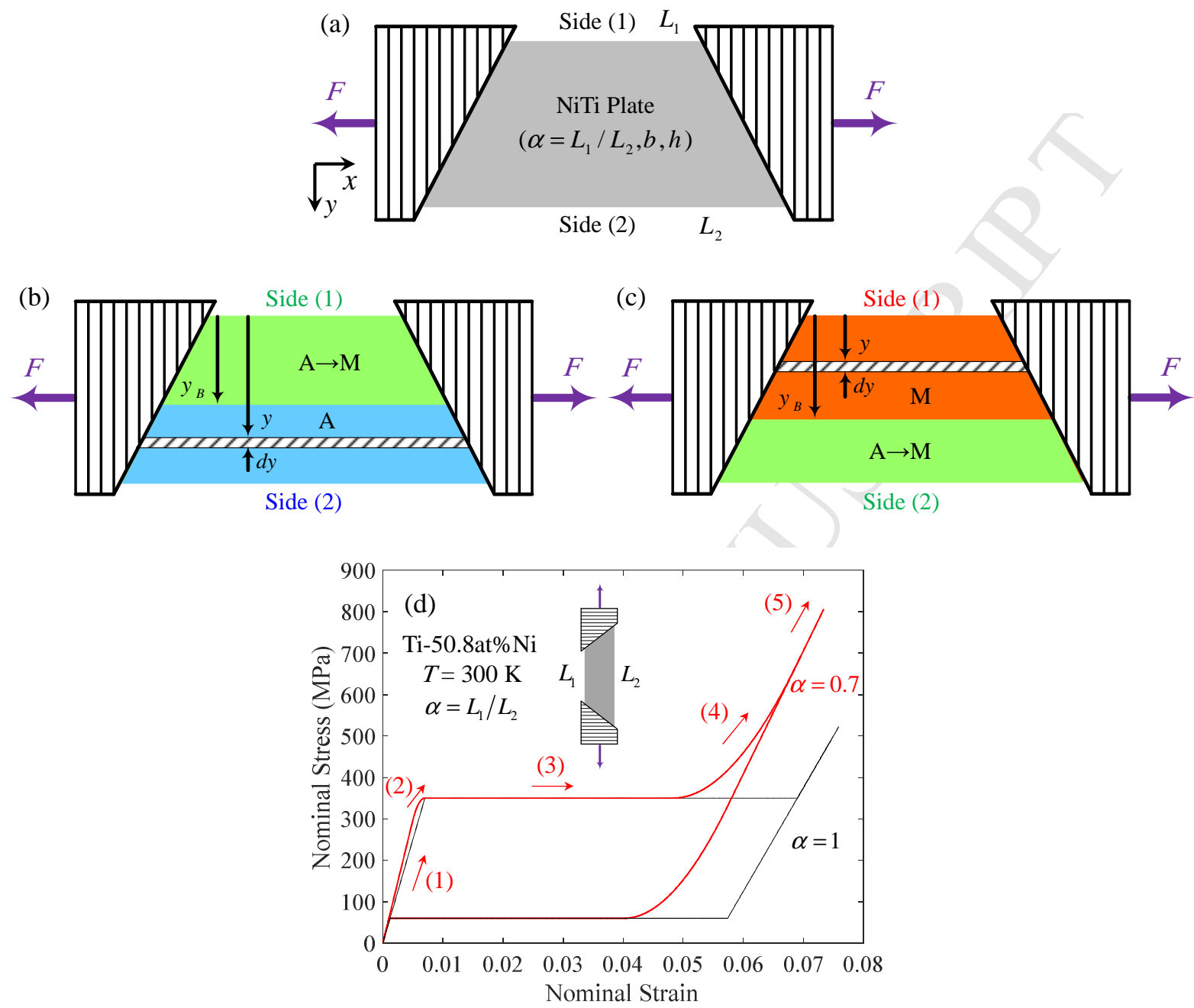

Fig. 6. Tapered plate design of the geometrically graded NiTi structure, (a): schematic drawing of the structure, (b): the initiation of transformation at stage (2), (c): the completion of transformation at stage (4), (d): the deformation behaviour for $\alpha=0.7$ and deformation stages.

Table 3. Stress-strain relationship of a tapered plate structure at different deformation stages.

\begin{tabular}{|l|l|l|}
\hline Stage & Nominal strain interval & Nominal stress-strain relationship \\
\hline 1 & $0 \leq \varepsilon<\alpha \frac{\sigma_{t}}{E_{A}}$ & $\sigma=\frac{E_{A} \ln \alpha}{\alpha-1} \varepsilon$ \\
\hline 2 & $\alpha \frac{\sigma_{t}}{E_{A}} \leq \varepsilon<\frac{\sigma_{t}}{E_{A}}$ & $\sigma=\frac{E_{A} \varepsilon}{1-\alpha}\left[1-\ln \left(\frac{E_{A} \varepsilon}{\sigma_{t}}\right)\right]-\frac{\alpha \sigma_{t}}{1-\alpha}$ \\
\hline
\end{tabular}




\begin{tabular}{|l|l|l|}
\hline 3 & $\frac{\sigma_{t}}{E_{A}} \leq \varepsilon<\alpha\left(\frac{\sigma_{t}}{E_{A}}+\varepsilon_{t}\right)$ & $\sigma=\sigma_{t}$ \\
\hline 4 & $\alpha\left(\frac{\sigma_{t}}{E_{A}}+\varepsilon_{t}\right) \leq \varepsilon<\frac{\sigma_{t}}{E_{A}}+\varepsilon_{t}$ & $\sigma=\sigma_{t}+\frac{E_{M} \varepsilon}{1-\alpha} \ln \left[\frac{\varepsilon}{\alpha\left(\frac{\sigma_{t}}{E_{A}}+\varepsilon_{t}\right)}\right]$ \\
& & $-\frac{E_{M}}{1-\alpha}\left[\varepsilon-\alpha\left(\frac{\sigma_{t}}{E_{A}}+\varepsilon_{t}\right)\right]$ \\
\hline 5 & $\varepsilon \geq \frac{\sigma_{t}}{E_{A}}+\varepsilon_{t}$ & $\sigma=\sigma_{t}-E_{M}\left(\frac{\sigma_{t}}{E_{A}}+\varepsilon_{t}\right)+\frac{E_{M} \varepsilon}{\alpha-1} \ln \alpha$ \\
& & \\
\hline
\end{tabular}

\section{Experimental evaluation}

Ti-50.8at\% Ni sheet of $0.1 \mathrm{~mm}$ thickness was used to fabricate geometrically graded NiTi samples in parallel configuration. Two types of samples were fabricated as per design (b)(II)-(i) and (b)-(II)-(ii) shown in Fig. 1, i.e., tapered plate samples and discrete parallel-strip samples. The width of the tapered plates was $7 \mathrm{~mm}$. For (b)-(II)-(ii), strips of $1.5 \mathrm{~mm}$ in width were cut from the sheet by electrical discharge machining. The maximum gauge length of samples was $30 \mathrm{~mm}$. Different length aspect ratios were used for both designs. Tensile testing was carried out using an Instron 5982 machine under displacement-control mode at a strain rate of $2 \times 10^{-4} / \mathrm{sec}$ with respect to the maximum length $\left(L_{2}\right)$. The testing temperature was $300 \mathrm{~K}$. Fig. 2(c) shows the tensile deformation behaviour of the alloy at $300 \mathrm{~K}$. The material showed good pseudoelastic behaviour with a flat stress plateau at $2350 \mathrm{MPa}$ over the forward $\mathrm{A} \rightarrow \mathrm{M}$ transformation and full transformation strain recovery upon reverse $\mathrm{M} \rightarrow \mathrm{A}$ transformation.

Fig. 7 shows the tensile experiments on several types of geometrically graded NiTi samples with parallel design configuration. Figs. 7(a)-(c) show also the comparison of the experimental results with the developed analytical modelling (by using material properties of Eq. (5)). It is evident that the analytical modelling can suitably predict the actual deformation behaviour of such structures. Fig 7(d) shows the tensile deformation behaviour of two-strip structures with different length aspect ratios. All samples exhibited partial stress gradient 
over stress-induced transformation. This figure confirms the analytical results presented in Fig. 4(c) regarding the effect of $\alpha$ variation on the deformation behaviour of two-strip structures. Also, it is seen that the sample with $\alpha=0.65$ exhibited a large stress widow of $420 \mathrm{MPa}$ over the forward transformation stage.
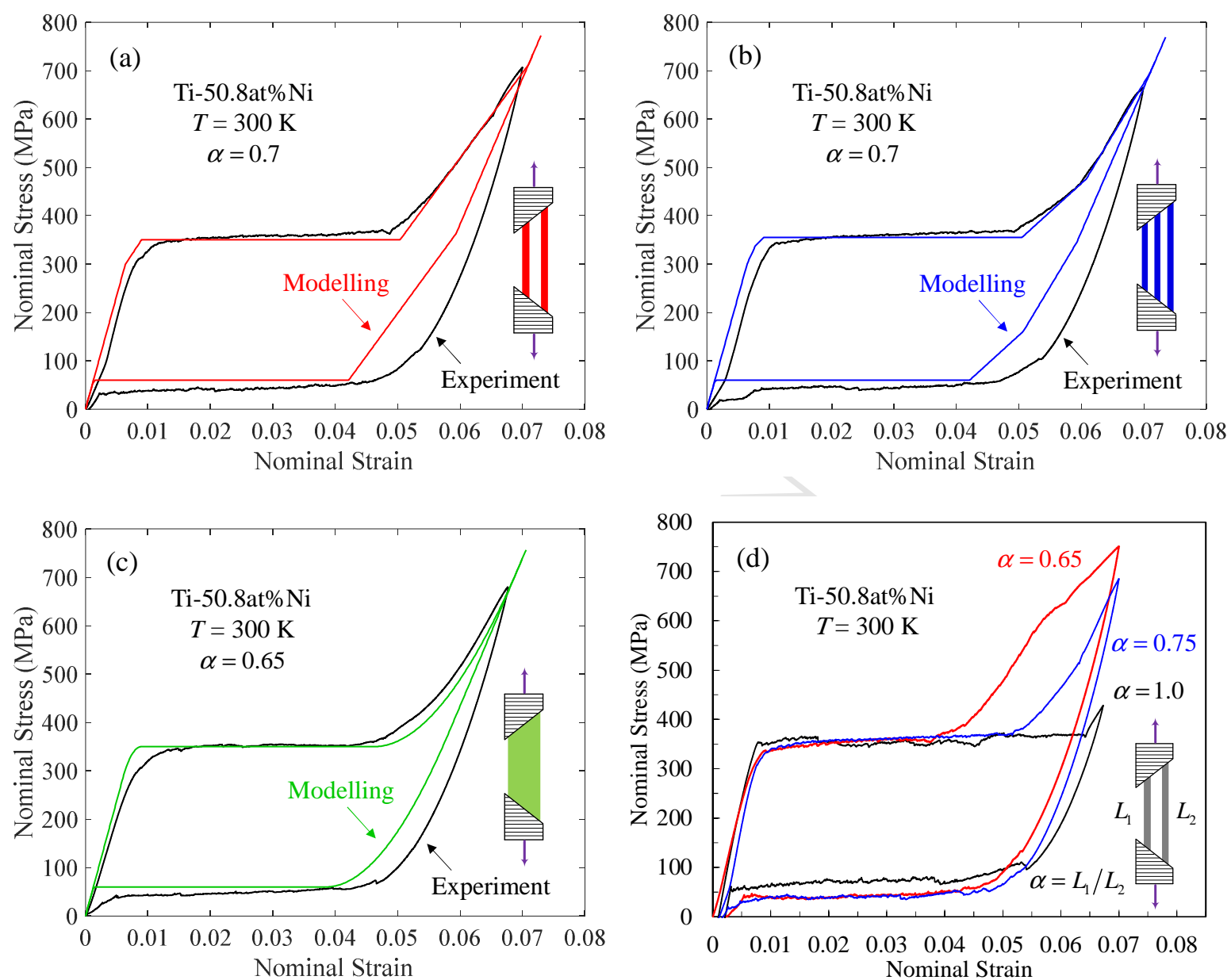

Fig. 7. Tensile experiments on geometrically graded NiTi samples with parallel design configuration and the prediction by the analytical modelling; (a): two-strip design with $\alpha=0.7$, (b): three strip design with $\alpha=0.7$, (c): tapered plate design with $\alpha=0.65$, (d): the effect of $\alpha$ variation on the deformation behaviour.

\subsection{Response comparison of parallel and series design configurations}

Fig. 8 shows the comparison of the response of geometrically graded structures with parallel and series design configurations [23, 25]. Fig. 8(a) shows the comparison of the global deformation behaviour of three Ti-50.8at\% Ni samples: a uniform strip (black curve), a twostrip structure with parallel design configuration (red curve), and a geometrically graded plate with series design configuration as per design concept of Fig. 1(a)-(II)-(i) (blue curve). The 
aspect ratio (length or width ratio) of both geometrically graded samples is 0.65 . It is seen that both geometrically graded structures exhibited stress gradient over $\mathrm{A} \rightarrow \mathrm{M}$ transformation with expanded stress window compared to that of a uniform NiTi structure. Although the sample with series design provided a more continuous gradient behaviour over transformation, the resulted stress window (130 MPa) was much less than that of the sample with parallel design (420 MPa) over $7 \%$ of nominal strain. Fig. 8(b) shows the variation of stress windows over forward transformation against the aspect ratio for the two types of geometrical gradient. It is seen that the achieved stress windows of parallel design configuration are larger than those of series design configuration for all aspect ratios.
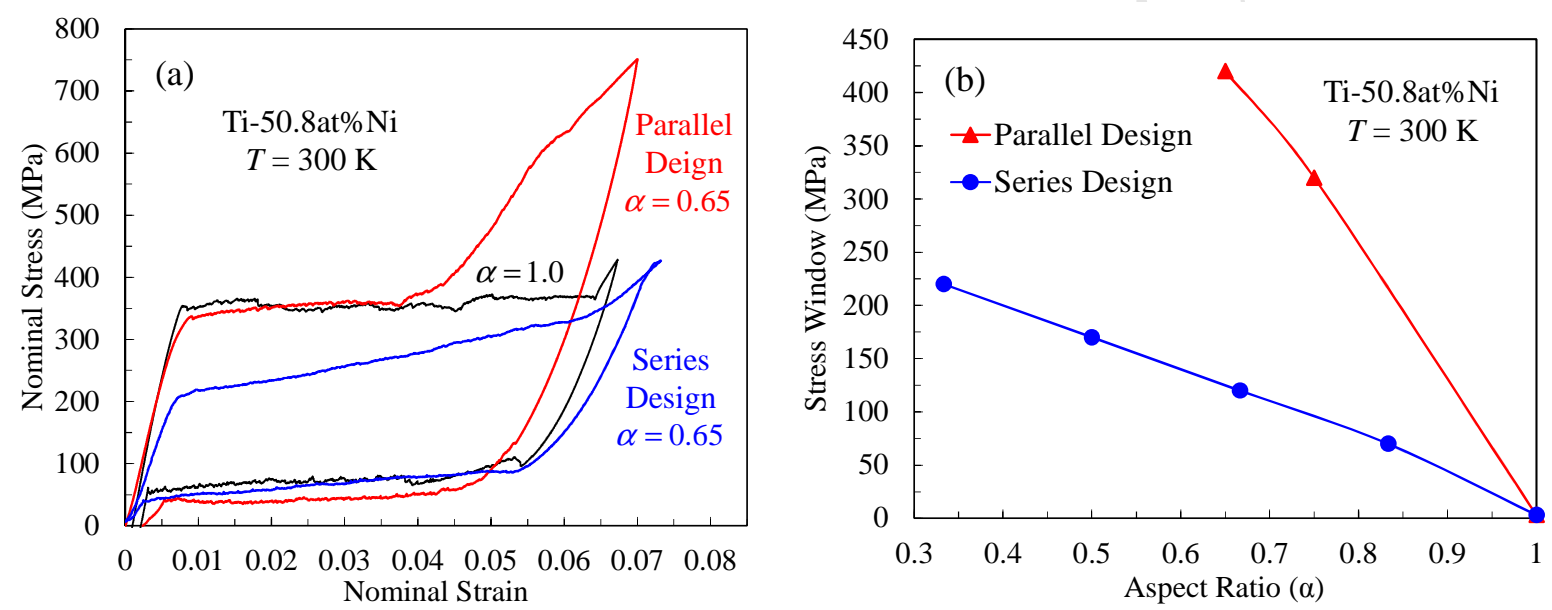

Fig. 8. Comparison of the parallel and series design configurations; (a): tensile deformation behaviour, (b): the variation of stress window versus aspect ratio.

\subsection{The effect of bending moment on transformation}

The varying distribution of force across the NiTi plate width, i.e. $y$-axis in Fig. 6(a), may create bending moment about the out of plane $z$-axis depending on the type of gripping mechanism. Here, we obtain the maximum value of the bending moment during $A \rightarrow M$ stress-induce transformation and estimate its effect on the overall deformation behaviour. As the length of the tapered plate of Fig. 6(a) is varying across the thickness according to Eq. (A1), the local strain decreases from side (1) to side (2) during loading. This yields in the variation of local force across the plate width with maximum on side (1) and minimum on side (2) during stage (1) of deformation. Considering Eq. (A1), the total bending moment about $z$-axis during stage (1) is obtained as 


$$
M_{z}=\frac{E_{A} \varepsilon h b^{2}}{\alpha-1}\left[1+\ln \alpha\left(\frac{\alpha}{1-\alpha}+\frac{1}{2}\right)\right]
$$

The bending moment increases by the increase of nominal strain $\varepsilon$ and reaches its maximum at the end of stage (1) as

$$
M_{\max }=\frac{\alpha h b^{2} \sigma_{t}}{\alpha-1}\left[1+\ln \alpha\left(\frac{\alpha}{1-\alpha}+\frac{1}{2}\right)\right]
$$

In stage (2), as the $\mathrm{A} \rightarrow \mathrm{M}$ transformation propagates from side (1) to side (2) and the local stress in the transforming layers remains constant, the bending moment decreases from the above maximum value by the increase of nominal strain and reaches zero at the end of this stage. In stage (3), the bending moment remains zero as the entire structure is in the course of martensitic transformation under constant stress.

Using Eq. (21), the maximum normal stress due to bending moment at the end of stage (1), which occurs at side (1), is obtained as

$$
\sigma_{b, \max }=\frac{6 \alpha \sigma_{t}}{\alpha-1}\left[1+\ln \alpha\left(\frac{\alpha}{1-\alpha}+\frac{1}{2}\right)\right]
$$

It is noted that the normal stress due to bending moment decreases from the above maximum value at side (1) to zero at the middle of the plate. Using Eq. (22) and considering $\alpha=0.7$, the average value of normal stress due to bending moment within the entire plate structure at the beginning of stage (2) is estimated as $\sim 0.07 \sigma_{t}$. The normal stress due to bending moment progressively decreases during stage (2) and becomes nil during stage (3). Considering the whole process of deformation during stress-induced transformation, the effect of possible occurrence of bending moment can be neglected in achieving the nominal stress-strain curve of the tapered NiTi plate shown in Fig. 6(d).

\subsection{Practicality of the proposed model}

It is known that polycrystalline NiTi exhibits strain localisation and propagation of deformation in macroscopic Lüders bands. The formation and propagation of these bands are characterised by a stress plateau in the alloy stress-strain curve [26]. In the current model, we consider the whole plateau length as the transformation strain and establish the nominal stress-strain relationship of the geometrically graded structure. Therefore, the localised 
deformation of the alloy is taken into account in obtaining the global deformation behaviour, even though the Lüders bands are not explicitly described by the model.

A critical factor, which limits the service life of SMAs including NiTi is functional fatigue. It has been reported that these alloys exhibit transformation-induced fatigue during thermal or mechanical loading cycles, which leads to the generation of macroscopic irrecoverable strain. It has been experimentally demonstrated that this behaviour is attributed to microstructural changes, e.g. generation of crystalline defects during repeated loading [27, 28]. The first step in predicting the response of SMAs is to develop constitutive models to describe the deformation mechanism of the alloy during tensile loading. The present study develops such model by obtaining closed-form solutions for stress-strain relationship of geometrically graded SMAs with parallel design configuration. It can be used as an engineering tool to design SMA structures with geometrical nonuniformity. The output from this model can later be implemented in numerical codes, such as finite element analysis packages [29-31], which take into account plastic deformation during cyclic loading, to study the fatigue behaviour of these structures.

\section{Conclusions}

(1) This article presents an analytical model to describe the deformation behaviour of geometrically graded shape memory alloys with parallel design configuration. Closedform solutions for nominal stress-strain relationships of such structures are derived. The analytical solutions satisfy well the experiments on pseudoelastic NiTi material.

(2) The geometrically graded structures with parallel design configuration show two main deformation stages over the forward stress-induced martensitic transformation, i.e., a flat stress plateau and a stress gradient with a positive slope. The gradient stress stage provides a stress window over transformation, which results in an improved actuation controllability. A stress window of $420 \mathrm{MPa}$ was achieved over the forward transformation for a sample with the length aspect ratio of 0.65 .

(3) By decreasing the length aspect ratio, the flat stress plateau length is decreased and the magnitude of the stress window and the stress-strain slope over the gradient stress stage are increased.

\section{Appendix A}


In this section, we establish the nominal stress-strain relationship of a tapered NiTi plate structure under uniaxial tensile loading as shown in Fig. 6(a) during sequential deformation stages. The length variation across the width of the plate is written as

$$
L(y)=L_{1}+\frac{y}{b}\left(L_{2}-L_{1}\right)
$$

Stage (1): $0 \leq \varepsilon<\alpha \frac{\sigma_{t}}{E_{A}}$

At this stage, the entire structure is in the austenite phase since the applied load is less than the critical value to induce martensitic transformation in side (1). The load $F$ is written as

$$
F=\int_{0}^{b} E_{A} \frac{\Delta L}{L(y)} h d y
$$

Using Eqs. (A1), (2), (3), and (A2), the nominal stress-strain relationship for this stage is obtained as

$$
\sigma=\frac{E_{A} \ln \alpha}{\alpha-1} \varepsilon
$$

Stage (2): $\alpha \frac{\sigma_{t}}{E_{A}} \leq \varepsilon<\frac{\sigma_{t}}{E_{A}}$

At this stage, the stress-induced martensitic transformation starts at side (1), where the stress has reached the forward transformation stress $\sigma_{t}$. As the loading level increases, the transformation progressively propagates towards side (2) as seen in Fig. 6(b). This stage ends when the transformation starts at side (2). During this stage, the locus of the start of the $\mathrm{A} \rightarrow \mathrm{M}$ transformation moves across the transverse direction from side (1) to side (2), with the region behind being partially transformed under the constant stress $\sigma_{t}$ (denoted $\mathrm{A} \rightarrow \mathrm{M}$ ) and that in front still in fully austenite state (denoted A). The boundary displacement is expressed by $y_{B}$, which varies from 0 to $b$. At the moment the $\mathrm{A} \rightarrow \mathrm{M}$ transformation starts at $y_{B}$, it can be written

$$
\sigma_{t}=E_{A} \frac{\Delta L}{L\left(y_{B}\right)}
$$

The load $F$ is divided into two parts carried by the partially transformed region and the fully austenite region: 


$$
F=F_{A \rightarrow M}+F_{A}=\sigma_{t} y_{B} h+\int_{y_{B}}^{b} E_{A} \frac{\Delta L}{L(y)} h d y
$$

Using Eqs. (A1), (2), (3), (A4), and (A5), the nominal stress-strain relationship for this stage is obtained as

$$
\sigma=\frac{E_{A} \varepsilon}{1-\alpha}\left[1-\ln \left(\frac{E_{A} \varepsilon}{\sigma_{t}}\right)\right]-\frac{\alpha \sigma_{t}}{1-\alpha}
$$

Stage (3): $\frac{\sigma_{t}}{E_{A}} \leq \varepsilon<\alpha\left(\frac{\sigma_{t}}{E_{A}}+\varepsilon_{t}\right)$

During this stage, the entire plate is in the course of martensitic transformation. Therefore, the total load $F$ remains unchanged. This stage ends when the transformation is completed at the shortest strip at side (1) while other parts of the plate are still in the course of transformation. The nominal stress of the structure during this stage is expressed as Eq. (12).

Stage (4): $\alpha\left(\frac{\sigma_{t}}{E_{A}}+\varepsilon_{t}\right) \leq \varepsilon<\frac{\sigma_{t}}{E_{A}}+\varepsilon_{t}$

This stage starts when side (1) is fully transformed to martensite and ends upon completion of transformation in the whole structure. Fig. 6(c) shows how transformation completes throughout the plate. As the loading level increases, the locus of the end of the transformation, defined by $y_{B}$, moves gradually from side (1) toward side (2). At the moment the $\mathrm{A} \rightarrow \mathrm{M}$ transformation completes at $y_{B}$, it can be written

$$
\sigma_{t}=E_{A}\left(\frac{\Delta L}{L\left(y_{B}\right)}-\varepsilon_{t}\right)
$$

The load $F$ is divided into two parts carried by the partially transformed region (denoted $\mathrm{A} \rightarrow \mathrm{M})$ and the fully martensite region (denoted $\mathrm{M})$ :

$$
F=F_{A \rightarrow M}+F_{M}=\sigma_{t}\left(b-y_{B}\right) h+\int_{0}^{y_{B}}\left[\sigma_{t}+E_{M}\left(\frac{\Delta L}{L(y)}-\frac{\sigma_{t}}{E_{A}}-\varepsilon_{t}\right)\right] h d y
$$

Using Eqs. (A1), (2), (3), (A7), and (A8), the nominal stress-strain relationship for this stage is obtained as 


$$
\sigma=\sigma_{t}+\frac{E_{M} \varepsilon}{1-\alpha} \ln \left[\frac{\varepsilon}{\alpha\left(\frac{\sigma_{t}}{E_{A}}+\varepsilon_{t}\right)}\right]-\frac{E_{M}}{1-\alpha}\left[\varepsilon-\alpha\left(\frac{\sigma_{t}}{E_{A}}+\varepsilon_{t}\right)\right]
$$

Stage (5): $\varepsilon \geq \frac{\sigma_{t}}{E_{A}}+\varepsilon_{t}$

At this stage, the entire structure has already transformed to martensite and is elastically stretched in the martensite phase. The load $F$ is written as

$$
F=\int_{0}^{b}\left[\sigma_{t}+E_{M}\left(\frac{\Delta L}{L(i)}-\frac{\sigma_{t}}{E_{A}}-\varepsilon_{t}\right)\right] h d y
$$

Using Eqs. (A1), (2), (3), and (A10), the nominal stress-strain relationship for this stage is obtained as

$$
\sigma=\sigma_{t}-E_{M}\left(\frac{\sigma_{t}}{E_{A}}+\varepsilon_{t}\right)+\frac{E_{M} \varepsilon}{\alpha-1} \ln \alpha
$$

Eqs. (A3), (A6), (12), (A9), and (A11) are the principal equations to describe the stress-strain behaviour of the tapered plate during loading. To define stress-strain relationships for the five stages of unloading, we can substitute $\sigma_{t}$ and $\varepsilon_{t}$ by $\sigma_{t}^{\prime}$ and $\varepsilon_{t}^{\prime}$ in the above equations and the corresponding strain intervals and use Eq. (4) to express the final relationships in terms of independent transformation parameters.

\section{Acknowledgement}

We wish to acknowledge the financial support to this work from the Australian Research Council in grants DE150101795 and DP160105066.

\section{References}

[1] J. Mohd Jani, M. Leary, A. Subic, M.A. Gibson, A review of shape memory alloy research, applications and opportunities, Mater. Design 56 (2014) 1078-1113.

[2] L. Sun, W.M. Huang, Z. Ding, Y. Zhao, C.C. Wang, H. Purnawali, C. Tang, Stimulusresponsive shape memory materials: A review, Mater. Design 33 (2012) 577-640. 
[3] N. Choudhary, D. Kaur, Shape memory alloy thin films and heterostructures for MEMS applications: A review, Sensors and Actuators A: Physical 242 (2016) 162-181.

[4] J. Van Humbeeck, Non-medical applications of shape memory alloys, Mater. Sci. Eng. A 273-275 (1999) 134-148.

[5] W. Huang, On the selection of shape memory alloys for actuators, Mater. Design 23(1) (2002) 11-19.

[6] M. Leary, S. Huang, T. Ataalla, A. Baxter, A. Subic, Design of shape memory alloy actuators for direct power by an automotive battery, Mater. Design 43 (2013) 460-466.

[7] N.B. Morgan, Medical shape memory alloy applications-the market and its products, Mater. Sci. Eng. A 378(1-2) (2004) 16-23.

[8] E.A. Williams, G. Shaw, M. Elahinia, Control of an automotive shape memory alloy mirror actuator, Mechatronics 20(5) (2010) 527-534.

[9] Y. Sutou, T. Omori, A. Furukawa, Y. Takahashi, R. Kainuma, K. Yamauchi, S. Yamashita, K. Ishida, Development of medical guide wire of Cu-Al-Mn-base superelastic alloy with functionally graded characteristics, Journal of Biomedical Materials Research Part B: Applied Biomaterials 69B(1) (2004) 64-69.

[10] Y. Facchinello, V. Brailovski, K. Inaekyan, Y. Petit, J.-M. Mac-Thiong, Manufacturing of monolithic superelastic rods with variable properties for spinal correction: Feasibility study, Journal of the Mechanical Behavior of Biomedical Materials 22(0) (2013) 1-11.

[11] P. Sedmák, J. Pilch, L. Heller, J. Kopeček, J. Wright, P. Sedlák, M. Frost, P. Šittner, Grain-resolved analysis of localized deformation in nickel-titanium wire under tensile load, Science 353(6299) (2016) 559-562.

[12] B.S. Shariat, Y. Liu, G. Rio, Pseudoelastic behaviour of perforated NiTi shape memory plates under tension, Intermetallics 50 (2014) 59-64.

[13] B.S. Shariat, Q. Meng, A.S. Mahmud, Z. Wu, R. Bakhtiari, J. Zhang, F. Motazedian, H. Yang, G. Rio, T.-h. Nam, Y. Liu, Functionally graded shape memory alloys: Design, fabrication and experimental evaluation, Mater. Design 124 (2017) 225-237.

[14] B.S. Shariat, Q. Meng, A.S. Mahmud, Z. Wu, R. Bakhtiari, J. Zhang, F. Motazedian, H. Yang, G. Rio, T.-h. Nam, Y. Liu, Experiments on deformation behaviour of functionally graded NiTi structures, Data in Brief 13 (2017) 562-568.

[15] Q. Meng, Y. Liu, H. Yang, B.S. Shariat, T.-h. Nam, Functionally graded NiTi strips prepared by laser surface anneal, Acta Mater. 60 (2012) 1658-1668. 
[16] Q. Meng, Z. Wu, R. Bakhtiari, B.S. Shariat, H. Yang, Y. Liu, T.-h. Nam, A unique "fishtail-like" four-way shape memory effect of compositionally graded NiTi, Scripta Mater. 127 (2017) 84-87.

[17] B.S. Shariat, Y. Liu, Q. Meng, G. Rio, Analytical modelling of functionally graded NiTi shape memory alloy plates under tensile loading and recovery of deformation upon heating, Acta Mater. 61(9) (2013) 3411-3421.

[18] B.S. Shariat, Y. Liu, G. Rio, Thermomechanical modelling of microstructurally graded shape memory alloys, J. Alloys Compd. 541 (2012) 407-414.

[19] B.S. Shariat, Y. Liu, G. Rio, Modelling and experimental investigation of geometrically graded NiTi shape memory alloys, Smart Mater. Struct. 22(2) (2013) 025030.

[20] B.S. Shariat, Y. Liu, G. Rio, Mathematical modelling of pseudoelastic behaviour of tapered NiTi bars, J. Alloys Compd. 577, Supplement 1 (2013) S76-S82.

[21] H. Liu, J. Wang, H.-H. Dai, Analytical study on stress-induced phase transitions in geometrically graded shape memory alloy layers. Part II: Analyses on geometrical shapes, loading procedures and boundary conditions, Mech. Mater. 112 (2017) 114-128.

[22] H. Liu, J. Wang, H.-H. Dai, Analytical study on stress-induced phase transitions in geometrically graded shape memory alloy layers. Part I: Asymptotic equation and analytical solutions, Mech. Mater. 112 (2017) 40-55.

[23] B.S. Shariat, Y. Liu, G. Rio, Hystoelastic deformation behaviour of geometrically graded NiTi shape memory alloys, Mater. Design 50 (2013) 879-885.

[24] Y. Liu, H. Yang, Strain dependence of the Clausius-Clapeyron relation for thermoelastic martensitic transformations in NiTi, Smart Mater. Struct. 16(1) (2007) S22-S27.

[25] B.S. Shariat, R. Bakhtiari, Y. Liu, Nonuniform transformation behaviour of NiTi in a discrete geometrical gradient design, J. Alloys Compd. 774 (2019) 1260-1266.

[26] P. Sittner, Y. Liu, V. Novak, On the origin of Lüders-like deformation of NiTi shape memory alloys, J Mech. Phys. Solids 53(8) (2005) 1719-1746.

[27] Y. Gao, L. Casalena, M.L. Bowers, R.D. Noebe, M.J. Mills, Y. Wang, An origin of functional fatigue of shape memory alloys, Acta Mater. 126 (2017) 389-400.

[28] P.K. Kumar, D.C. Lagoudas, Introduction to shape memory alloys, in: D.C. Lagoudas (Ed.), Shape memory alloys: modeling and engineering applications, Springer, 2008.

[29] R. Bakhtiari, B.S. Shariat, F. Motazedian, Z. Wu, J. Zhang, H. Yang, Y. Liu, Complex transformation field created by geometrical gradient design of NiTi shape memory alloy, Functional Materials Letters 10(01) (2017) 1740011. 
[30] B.S. Shariat, Y. Liu, G. Rio, Finite element computational modelling and experimental investigation of perforated NiTi plates under tension, Mater. Res. Bull. 48(12) (2013) 50995104.

[31] B.S. Shariat, Y. Liu, G. Rio, Numerical modelling of pseudoelastic behaviour of NiTi porous plates, J. Intell. Mater. Syst. Struct. 25(12) (2014) 1445-1455. 
- Closed-form stress-strain relationships were obtained for optimizing shape memory performance

- Transformation propagation in parallel design configuration was perpendicular to the loading axis

- Geometrically graded NiTi demonstrated partial stress gradient over transformation

- Widened transformation stress window for actuation application was created 